\title{
QLF-D: A Contemporary Plaque Control Tool in Children
}

\author{
Ratika Solanki ${ }^{1}$, Avantika Tuli ${ }^{2}$, Preeti Dhawan ${ }^{3}$, Nitin Khanduri ${ }^{4}$, Aditi Singh ${ }^{5}$
}

\begin{abstract}
Background and objectives: The main causative factors for maximum periodontal diseases are dental plaque and oral biofilms. This study was done to check the impact of quantitative light-induced fluorescence-digital (QLF-D) as a motivational tool for plaque control among children from various schools situated in Dehradun district along with education using audiovisual aids.

Materials and methods: A total sample of 800 school-going children including both males and females aged 6 to 12 years from various schools situated in Dehradun district of Uttarakhand were surveyed. A pro forma was prepared and the demographics of the students were noted. All the students were first examined for the Oral Hygiene Index-Simplified (OHI-S) and then the random division was done into two groups, group $\mathrm{A}$ (Q-scan) and group B (basic diagnostic aids) for examination of plaque index.
\end{abstract}

Results: The results of the study revealed that good OHI-S scores increased significantly from 1st visit to 3rd visit. The plaque scores for the control group, when examined with basic diagnostic aids of all three visits, were significantly good which increased subsequently from 1 st visit to 3rd visit. Good plaque index score increased significantly for the test group from 1st to 3rd visit when examined with QLF-D.

Conclusion: We found in our study that QLF technology is of paramount importance in epidemiological surveys and plays a pivotal role in evaluating masses in maintaining oral health care.

Keywords: Audiovisual aids, Dental plaque, Mechanical plaque control, Oral health education, Q-scan device.

International Journal of Clinical Pediatric Dentistry (2021): 10.5005/jp-journals-10005-2001

\section{INTRODUCTION}

The main causative factors for maximum periodontal diseases are dental plaque and oral biofilms. ${ }^{1}$ Conventional aids for plaque control require manual dexterity and are time-consuming whereas recent advancements are more accurate and less time-consuming. ${ }^{2}$ Brushing and flossing are practices to maintain good oral health, along with regular dental visits. ${ }^{3}$

There are various measures to evaluate plaque existence available for daily toothbrushing at home. The most common and inexpensive approach is plaque disclosing products. Disclosing solutions cause staining of teeth as it consists of phloxine $B, a$ staining substance widely used as a color additive for food, drugs, and cosmetics. ${ }^{4}$ Thus, to overcome these limitations, computerized dental plaque quantification has been attempted as it mainly focuses on the plaque covering the tooth surface. ${ }^{5}$

Oral health education has been considered a primary method via which dental services can provide oral health information and improve the health literacy of patients, especially when the health sources are finite. ${ }^{6,7}$ Schools are appropriate for applying for health education programs as it can be used to promote healthy lifestyles and self-care practices at a very low cost. ${ }^{8-10}$ School-based oral health education programs have produced positive outcomes in oral cleanliness, oral health knowledge, and oral behavior. ${ }^{11}$

Audiovisual aids are valuable tools in the field of health education due to their long-lasting impression on the target population. Studies utilizing audiovisual aids for improving oral health concluded them to have a great impact on oral health knowledge of the masses. ${ }^{12,13}$

Quantitative light-induced fluorescence (QLF) technology is commonly used in dentistry for the assessment of dental plaque. ${ }^{14}$ It is a portable device that can be used by the clinician chairside to visualize plaque and precarious lesions ${ }^{15}$ (Fig. 1). Education using
1,2,4,5 Department of Pediatric and Preventive Dentistry, Seema Dental College and Hospital, Rishikesh, Uttarakhand, India

${ }^{3}$ Department of Pedodontics and Preventive Dentistry, BRS Dental College, Panchkula, Haryana, India

Corresponding Author: Ratika Solanki, Department of Pediatric and Preventive Dentistry, Seema Dental College and Hospital, Rishikesh, Uttarakhand, India, Phone: +91 9690993388, e-mail: 9690993388ratika@gmail.com

How to cite this article: Solanki R, Tuli A, Dhawan P, et al. QLF-D: A Contemporary Plaque Control Tool in Children. Int J Clin Pediatr Dent 2021;14(4):502-505.

Source of support: Nil

Conflict of interest: None

QLF technology seems to be effective in changing the attitude of children as they can visualize the presence of plaque in their mouth which serves as a better educational guide. ${ }^{16}$

A benefit of using QLF-D may be in its ability to motivate patient behavior. Hence, the purpose of this study was to evaluate the QLF device as a vector to improve the perception of schoolchildren regarding oral health care.

\section{Materials and Methods}

\section{Source of the Data}

A total sample of 800 school-going children including both males and females aged 6 to 12 years from various schools situated in the Dehradun district of Uttarakhand were assessed. Informed consent was obtained from the participant's parents, who were provided with detailed information on the study protocol and institutional ethical clearance was acquired from the Ethical committee. 


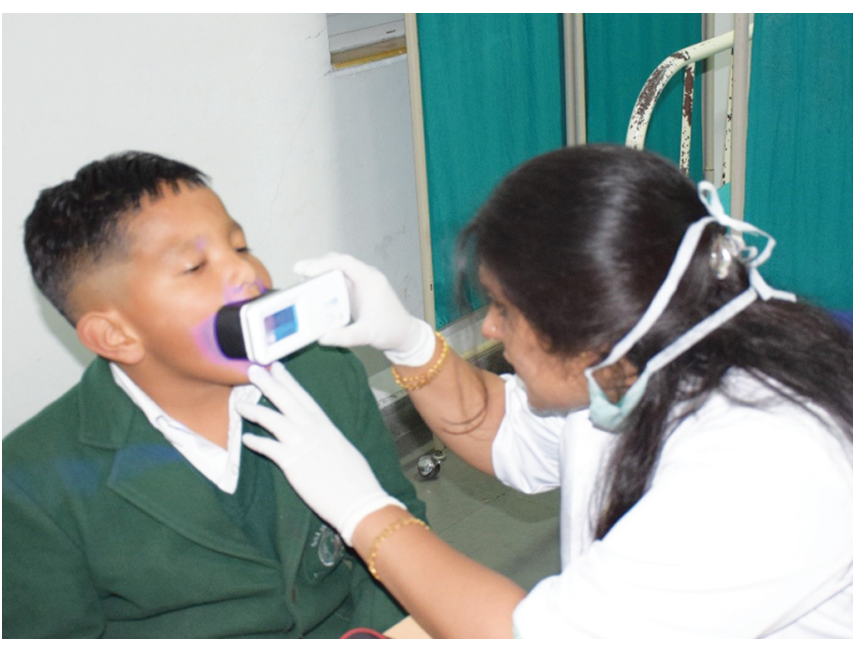

Fig. 1: Q-Scan device used in the study

\section{Inclusion Criteria}

- Children in the age-group of 6 to 12 years with mixed dentition.

- Cooperative children.

- Children with no medical history of systemic diseases/disorders.

\section{Exclusion Criteria}

- Children with serious medical illnesses (psychiatric problems, endocrine diseases).

- Children below the age of 6 years and above the age of 12 years.

- Children with disabilities.

\section{Method of Collection of Data}

All the students were first examined for the Oral Hygiene IndexSimplified (OHI-S). Then, the random division of the group was done.

- The test group, i.e., group I which was clinically assessed through the Q-scan device for plaque index (Fig. 2).

- The control group, i.e., group II which was clinically checked for plaque index with the help of basic diagnostic aids.

The oral hygiene levels of the children in the study were examined using the OHI-S (Green and Vermillion in 1960) ${ }^{17}$ and the plaque score was measured using the plaque index (Silness and Loe in 1964). ${ }^{18}$ After the examination, a health talk was given to the students and teachers of the respective schools, with the help of audiovisual aids along with a demonstration of proper toothbrushing techniques after the completion of data assembled.

All participants were examined using torchlight, dental mirrors, and mouth probes. The OHI-S index was used to assess the oral hygiene score for six index teeth: \#11, \#16, \#26, \#31, \#36, and \#46. The plaque levels were evaluated using plaque index for six index teeth: \#12, \#16, \#24, \#32, \#36, and \#44.

The Q-scan device was used to help students visualize plaque in their mouth in red color and the importance of its daily removal.

All participants were asked questions regarding their oral health, attitude, and dental health practices. Questions were

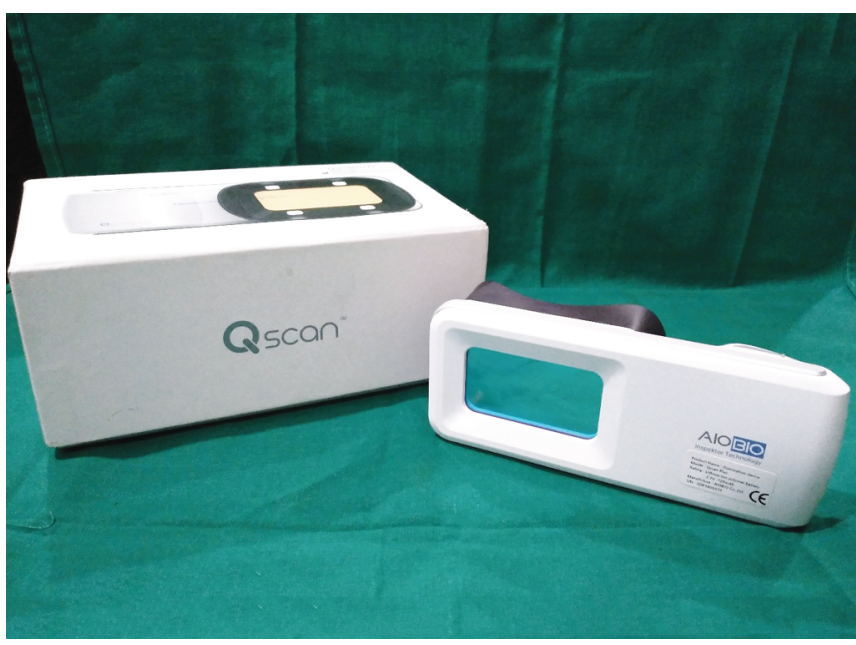

Fig. 2: Examination for plaque index using Q-scan device

included on brushing habits, aids used for brushing, frequency, and rinsing habits to assess the practices related to oral health.

Lessons on oral hygiene were taught to both the groups in a traditional lecture format lasting 10 minutes along with a video presentation and also demonstrational models.

The OHI-S index and the plaque index assessment were repeated after a period of 1 and 3 months to check for the impact of the educational program on the oral hygiene index status and also to check the effectiveness of the Q-scan device on the plaque index of the target population. The Statistical Package for Social Sciences (SPSS) version 25.0 and Med Calc software were used for statistical analysis. The statistical tests used were repeated measures analysis of variance (ANOVA) test, post hoc tests, and unpaired or independent $t$-test.

\section{Results}

The distribution of the OHI-S index score was compared between the 1st, 2nd, and 3rd visit using the Chi-square test. Good OHI-S index score increased significantly from 1st to 2 nd to 3 rd visit, thus signifying the importance of an oral health awareness program using AV Aids. Also, it was observed that in all three visits, the maximum participants exhibited a fair OHI-S score ( $p$ value $=$ 0.001) (Table 1).

The distribution of the plaque index score was compared for the control group which was examined with basic diagnostic aids between 1st, 2nd, and 3rd visit using the Chi-square test. Good plaque index score increased significantly from 1st to 2 nd to 3 rd visit, respectively ( $p$ value $=0.006$ ) (Table 2 ).

The distribution of the plaque index score was compared between the 1st, 2nd, and 3rd visits using the Chi-square test. Good plaque index score increased significantly from 1 st to 2 nd to 3 rd visit, thus proving the impact of Q-scan device as a motivational tool for plaque control ( $p$ value $=0.001)($ Table 3$)$.

\section{Discussion}

Dental plaque plays a dominant role in the causation of both dental caries and periodontal disease. Supragingival plaque is exposed to saliva and to the natural self-cleansing mechanism existing in the oral cavity which may eliminate food debris, but do not adequately 
QLF-D: The Need of the Hour

Table 1: Intercomparison of OHI-S score between all three visits

\begin{tabular}{|c|c|c|c|c|c|c|}
\hline \multirow[b]{2}{*}{ OHI-S index } & \multicolumn{2}{|c|}{1 st visit } & \multicolumn{2}{|c|}{ 2nd visit } & \multicolumn{2}{|c|}{$3 r d$ visit } \\
\hline & Frequency & Percent & Frequency & Percent & Frequency & Percent \\
\hline Good & 5 & 0.6 & 9 & 1.1 & 70 & 8.8 \\
\hline Fair & 573 & 71.6 & 643 & 80.4 & 700 & 87.5 \\
\hline Poor & 222 & 27.8 & 148 & 18.5 & 30 & 3.8 \\
\hline Total & 800 & 100.0 & 800 & 100.0 & 800 & 100.0 \\
\hline \multicolumn{7}{|c|}{$\chi^{2}$ value $=11.285, p$ value $=0.001^{*}$} \\
\hline
\end{tabular}

Chi-square test

*Significant difference

Table 2: Intercomparison of plaque scores for the control group between the three visits

\begin{tabular}{|c|c|c|c|c|c|c|}
\hline \multirow{2}{*}{$\begin{array}{l}\text { Control group } \\
\text { Plaque index at } \\
\text { 1st visit }\end{array}$} & \multicolumn{2}{|c|}{ Plaque index at 1st visit } & \multicolumn{2}{|c|}{ Plaque index at $2 n d$ visit } & \multicolumn{2}{|c|}{ Plaque index at 3rd visi } \\
\hline & Frequency & Percent & Frequency & Percent & Frequency & Percent \\
\hline Good & 253 & 63.3 & 316 & 79.0 & 364 & 91.0 \\
\hline Fair & 146 & 36.5 & 84 & 21.0 & 36 & 9.0 \\
\hline Poor & 1 & 0.3 & 0 & 0.0 & 0 & 0.0 \\
\hline$\chi^{2}$ value $=7.485$ & value $=0.00$ & & & & & \\
\hline
\end{tabular}

Chi-square test

*Significant difference

Table 3: Intercomparison of plaque score for the Q-scan group between all three visits

\begin{tabular}{|c|c|c|c|c|c|c|}
\hline \multirow[b]{2}{*}{ Test group } & \multicolumn{2}{|c|}{ Plaque index at 1st visit } & \multicolumn{2}{|c|}{ Plaque index at 2nd visit } & \multicolumn{2}{|c|}{ Plaque index at 3rd visi } \\
\hline & Frequency & Percent & Frequency & Percent & Frequency & Percent \\
\hline Good & 266 & 66.5 & 366 & 91.5 & 386 & 96.5 \\
\hline Fair & 124 & 31.0 & 34 & 8.5 & 14 & 3.5 \\
\hline Poor & 10 & 2.5 & 0 & 0.0 & 0 & 0.0 \\
\hline \multicolumn{7}{|c|}{$\chi^{2}$ value $=9.852, p$ value $=0.001^{*}$} \\
\hline
\end{tabular}

Chi-square test

*Significant difference

remove dental plaque. Thus, regular oral hygiene practice is a requisite for proper plaque elimination. ${ }^{19}$

In the current study, also children were educated about proper oral hygiene measures through a health talk wherein they were told about the importance of oral health and brushing and it was concluded from the results that school-based oral health awareness programs had a great impact on the sample population. A similar study was conducted on a school-based oral health education program using experimental learning (EL) or traditional lecturing (TL) in adolescents and it was found that the EL program was more effective than the TL program and it also helped in improving knowledge about oral health, attitude, attendance, and dental habits. ${ }^{20}$ Similarly, a study was conducted to check the impact of the oral health awareness program on children and it had a positive effect on the oral health care of children. ${ }^{21}$

In the present study, also the total sample of 800 was educated for oral hygiene and proper brushing technique with the help of AV aids which had key educational messages regarding oral health and from the results, it was concluded that education with AV aids proved to have a significant impact on oral hygiene levels of the masses. This is consistent with a similar study which concluded that $\mathrm{AV}$ aids help in providing anticipatory guidance regarding infant oral health in high-risk populations along with a comprehensive, self-directed, evidence-based approach to the promotion of infant oral health. ${ }^{12}$

Quantitative light-induced fluorescence appears to be a technique that has many uses in dentistry. It has been validated both qualitatively and quantitatively for the assessment of caries. Based on a similar study, it was confirmed that QLF-D also aids in measuring old dental plaques that have strong clinical relation to diseases of the oral cavity. ${ }^{20}$ In the present study, it was seen that oral health education using QLF technology improved the oral hygiene and plaque status of children. This is also in accordance with a study which reported that QLF could be useful for plaque detection and encouraging children to receive daily habits and oral hygiene instructions. ${ }^{21}$ So, this is how it was proved in this study how the Q-scan device turned out to be a better motivational tool for plaque control in school-going children.

\section{Conclusion}

Education that utilizes new technologies and their associated devices can also be expected to be more effective. This study also reflected the importance of additional equipment such as the 
Q-scan device in oral health education can yield better educational effects than the traditional education system. Also, QLF can be used as a motivation booster for high caries risk individuals who are difficult to motivate. Thus, education using QLF technology should be included in the school health curriculum.

\section{References}

1. Axelsson P, Albandar JM, Rams TE. Prevention and control of periodontal diseases in developing and industrialized nations. Periodontol 2000 2002;29(1):235-246. DOI: 10.1034/j.16000757.2002.290112.x.

2. Mandal A, Singh DK, Siddiqui $H$, et al. New dimensions in mechanical plaque control: an overview. Indian J DentSci 2017;9(2):133-139. DOI: 10.4103/IJDS.IJDS_18_17.

3. Ahad M, Gheena S. Awareness of tooth brushing techniques and proper oral hygiene among school children. J Pharmaceut Sci Res 2015;7(6):367-372.

4. Coulthwaite L, Pretty IA, Smith PW, et al. The microbiological origin of fluorescence observed in plaque on dentures during QLF analysis. Caries Res 2006;40(2):112-116. DOI: 10.1159/000091056.

5. Staudt CB, Kinzel S, Hassfeld S, et al. Computer-based intraoral image analysis of the clinical plaque removing capacity of 3 manual toothbrushes. J Clin Periodontol 2001;28(8):746-752. DOI: 10.1034/j.1600-051x.2001.280805.x.

6. Kwan SY, Petersen PE, Pine CM, et al. Health-promoting schools: an opportunity for oral health promotion. World Health Organ 2005;83(9):677-685. DOI: /S0042-96862005000900013.

7. Redmond CA, Blinkhorn FA, Kay EJ, et al. A cluster randomized controlled trial testing the effectiveness of a school-based dental health education program for adolescents. J Public Health Dent 1999;59(1):12-17. DOI: 10.1111/j.1752-7325.1999.tb03229.x.

8. Maes L, Lievens J. Can school make a difference? A multilevel analysis of children's risk and health behaviour. J Soc Sci Med 2003;56(3):517529. DOI: 10.1016/s0277-9536(02)00052-7.

9. Walsh MM. Effects of school-based dental health education on knowledge, attitudes and behaviour of adolescents in San Francisco,. Community Dent Oral Epidemiol 1985;13(3):143-147. DOI: 10.1111/ j.1600-0528.1985.tb00430.x.
10. Alsada LH, Sigal MJ, Limeback $H$, et al. Development and testing of an audio-visual aid for improving infant oral health through primary caregiver education. J Can Dent Assoc 2005;71(4):241.

11. Rothe V, Kebriaei A, Pitner S, et al. Effectiveness of a presentation on infant oral health care for parents. Int J Pediatr Dent 2010;20(1):37-42. DOI: 10.1111/j.1365-263X.2009.01018.x.

12. Lussi A. Comparison of different methods for the diagnosis of fissure caries without cavitation. Caries Res 1993;27(5):409-416. DOI: 10.1159/000261572.

13. Hope $\mathrm{CK}$, Wang Q, Burnside G, et al. Assessing the association between oral hygiene and preterm birth by quantitative light-induced fluorescence. Scienti World J 2014;10:1-10. DOI: 10.1155/2014/374694.

14. Askelson NM, Chi DL, Momany ET, et al. Encouraging early preventive dental visits for preschool-aged children enrolled in medicaid: using the extended parallel process model to conduct formative research. J Public Health Dent 2014;74(1):64-70. DOI: 10.1111/j.17527325.2012.00369.x.

15. Greene JC, Vermillion JR. The simplified oral hygiene index. J Am Dent Assoc 1964;68(1):7-13. DOI: 10.14219/jada.archive.1964.0034.

16. Menon L, Ramamurthy J. New vistas in plaque control. IOSR J Dent Med Sci 2014;13(3):64-68. DOI: 10.9790/0853-13356468.

17. Angelopoulou MV, Oulis CJ, Kavvadia K. School-based oral healtheducation program using experiential learning or traditional lecturing in adolescents: a clinical trial. Int Dent J 2014;64(5):278-284. DOI: 10.1111/idj.12123.

18. Priya PR, Asokan S, Janani RG, et al. Effectiveness of school dental health education on the oral health status and knowledge of children: a systematic review. Indian J Dent Res 2019;30(3):437-449. DOI: 10.4103/ijdr.IJDR_805_18.

19. Jain SD, Jain M, Khan $\mathrm{K}$, et al. Evaluating the use of audio-visual aids in knowledge gained among patients visiting a dental college in Greater Noida. Int Healthcare Res J 2017;10(9):278-283. DOI: 10.26440/ IHRJ/01_09/131.

20. Jong EJ, Higham SM, Smith PW, et al. Quantified light-induced fluorescence, review of a diagnostic tool in prevention of oral disease. J Appl Phys 2009(1):105. DOI: 10.1007/s00340-009-3412-4.

21. Watanabe K, Sasabe T, Nakamura A, et al. Advantage of introducing quantitative light induced fluorescence in school dental check-ups. Health 2018;10(08):1095-1106. DOI: 10.4236/health.2018.108083. 